\title{
HYSTERESIS SUPPRESSION IN NONLINEAR MATHIEU M/NEMS RESONATORS
}

\author{
N. Kacem \\ S. Hentz \\ CEA/Leti - MINATEC \\ Microsystems Components Laboratory \\ Grenoble 38054 France \\ Email: najib.kacem@cea.fr \\ Email: sebastien.hentz@cea.fr
}

\author{
S. Baguet \\ R. Dufour \\ Université de Lyon, CNRS \\ Insa Lyon LaMCoS CNRS UMR5259 \\ Villeurbanne F69621 France \\ Email: sebastien.baguet@insa-lyon.fr \\ Email: regis.dufour@insa-lyon.fr
}

\section{ABSTRACT}

In order to compensate the loss of performances when scaling resonant sensors down to NEMS, it proves extremely useful to study the behavior of resonators up to very high displacements and hence high non linearities. This work describes a comprehensive non linear multiphysics model based on the EulerBernoulli equation which includes both mechanical and electrostatic nonlinearities valid up to displacements comparable to the gap in the case of a capacitive doubly clamped beam. Moreover, the model takes into account the fringing field effects, significant for thin resonators. The model has been compared to electrical measurements of devices fabricated on $200 \mathrm{~mm}$ SOI wafers and show a very good agreement. This model allows designers to cancel out non linearities by tuning some design parameters and thus gives the possibility to drive the resonator beyond its critical amplitude. Consequently, the sensors performances can be enhanced to the maximum bellow the pull-in amplitude, while keeping a linear behavior.

\section{Introduction}

Nanoelectromechanical systems (NEMS) have been the focus of recent applied and fundamental research. With critical dimensions down to a few tens of nm, most NEMS are resonant devices. In this size regime, they display high fundamental resonance frequencies (recently going beyond $1 \mathrm{GHz}[1,2]$ ), diminished active masses, tolerable force constants and relatively high quality factors in the range of $10^{2}-10^{4}$. These attributes collectively make NEMS suitable for a multitude of technological applications such as ultrasensitive force and mass sensing, narrow band filtering, and time keeping. However, the smaller the structures, the sooner nonlinearities occur [3], reducing their dynamic range and even making them extremely difficult to detect, as proves the abundant literature about characterization techniques [4]. The sensitivity of a resonant sensor may be given by its frequency noise spectral density [5]:

$$
S_{\omega}(\omega)=\left(\frac{\omega_{n}}{2 Q}\right)^{2} \frac{S_{x}\left(\omega_{n}\right)}{P_{0}}
$$

Where $S_{x}\left(\omega_{n}\right)$ is the displacement spectral density and $P_{0}$ is the displacement carrier power, ie the RMS drive amplitude of the resonator $\frac{1}{2} A^{2}$. The latter is classically driven below the hysteretic limit (critical amplitude) due to the mechanical nonlinearity [3]. Following Postma et al. [3], the resonator open-loop stability amplitude is $A_{c} \propto \frac{h}{Q}$ where $h$ is the resonator vibrating thickness and $Q$ is its quality factor. It is easy to see how drastic the performance degradation may be in the case of a NEMS with small $\mathrm{h}$. It has been shown that closed-loop control permits operating beyond the critical amplitude [6]), eventually up to the pull-in amplitude in the case of capacitive transduction. But to do so, it is necessary -first, to precisely know this dynamic pullin amplitude, and -second, to avoid the noise aliasing issue [7], so as not to degrade the amplitude noise density. 
To this end, the non linear behavior of resonators has to be explored, and numerous models have been presented. Some of them are purely analytical [8-10] but they include coarse assumptions concerning nonlinearities. For example, Kozinsky et al. [10] use a nonlinear model with a 3rd order Taylor series expansion of the electrostatic forcing applied to a nanoresonator in order to tune the effective Duffing coefficient using an external electrostatic potential. However, this approximation is limited by the beam displacement (not more than $20 \%$ of the gap), and the static displacement is used for tuning, which stays limited.

Other models $[11,12]$ are more complicated but use numerical integrations (shooting and continuation) which make them less interesting for M/NEMS designers.

In this paper, a compact and analytical model including all main sources of nonlinearities is presented and validated thanks to the characterization of a vibrating accelerometer sensing element, an electrostatically driven clamped-clamped beam. It is shown how the model can be used to engineer the resonator non-linearities in order to drive it at the highest possible amplitude while suppressing its hysteresis, thus retrieving a linear behaviour.

\section{Model}

A clamped-clamped microbeam is considered (Figure 1)

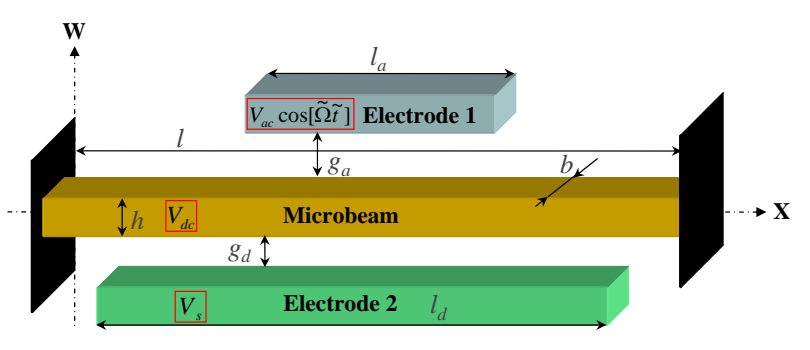

Figure 1. Schema of an electrically actuated microbeam

\section{Equation of motion}

The transverse deflection of the microbeam $w(x, t)$ is governed by the nonlinear Euler-Bernoulli equation, which is the commonly used equation of motion for a slender beam

$$
E I \frac{\partial^{4} \tilde{w}}{\partial \tilde{x}^{4}}+\rho b h \frac{\partial^{2} \tilde{w}}{\partial \tilde{t}^{2}}+\tilde{c} \frac{\partial \tilde{w}}{\partial \tilde{t}}-\left[\tilde{N}+\frac{E b h}{2 l} \int_{0}^{l}\left[\frac{\partial \tilde{w}}{\partial \tilde{x}}\right]^{2} d \tilde{x}\right] \frac{\partial^{2} \tilde{w}}{\partial \tilde{x}^{2}}
$$

$$
\begin{gathered}
=\frac{1}{2} \varepsilon_{0} \frac{b C_{n 1}[\operatorname{Vac} \cos (\tilde{\Omega} \tilde{t})-V d c]^{2}}{\left(g_{a}-\tilde{w}\right)^{2}} H_{1}(\tilde{x}) \\
-\frac{1}{2} \varepsilon_{0} \frac{b C_{n 2}[V s-V d c]^{2}}{\left(g_{d}+\tilde{w}\right)^{2}} H_{2}(\tilde{x})
\end{gathered}
$$

With:

$$
\begin{aligned}
& H_{1}(\tilde{x})=H\left(\tilde{x}-\frac{l+l_{a}}{2}\right)-H\left(\tilde{x}-\frac{l-l_{a}}{2}\right) \\
& H_{2}(\tilde{x})=H\left(\tilde{x}-\frac{l+l_{d}}{2}\right)-H\left(\tilde{x}-\frac{l-l_{d}}{2}\right)
\end{aligned}
$$

where $\tilde{x}$ is the position along the microbeam length, $E$ and $I$ are the Young's modulus and moment of inertia of the cross section. $\tilde{N}$ is the applied tensile axial force due to the residual stress on the silicon (or the effect of the measurand in the case of an accelerometer), $\tilde{t}$ is time, $\rho$ is the material density, $h$ is the microbeam thickness, $g_{a}$ and $g_{d}$ are respectively the actuation and the sensing capacitor gap width, $\varepsilon_{0}$ is the dielectric constant of the gap medium. The last term in Equation (2) represents an approximation of the electric force assuming a resonator design with 2 stationary electrodes : electrode 1 for the actuation and electrode 2 for the sensing including the fringing field effect using the coefficients $C_{n i}$. Since the electrodes do not act on the whole length of the beam, the electrostatic force distributions are modeled by means of Heaviside functions $H(\tilde{x})$. The boundary conditions are:

$$
\tilde{w}(0, \tilde{t})=\tilde{w}(l, \tilde{t})=\frac{\partial \tilde{w}}{\partial \tilde{x}}(0, \tilde{t})=\frac{\partial \tilde{w}}{\partial \tilde{x}}(l, \tilde{t})=0
$$

\section{Normalization}

For convenience and equations simplicity, let the following nondimensional variables be introduced:

$$
w=\frac{\tilde{w}}{g_{d}}, x=\frac{\tilde{x}}{l}, t=\frac{\tilde{t}}{\tau}, R_{g}=\frac{g_{a}}{g_{d}}
$$

where $\tau=\frac{2 l^{2}}{h} \sqrt{\frac{3 \rho}{E}}$.

Substituting Equation (6) into Equations (2) and (5) yields

$$
\begin{array}{r}
\frac{\partial^{4} w}{\partial x^{4}}+\frac{\partial^{2} w}{\partial t^{2}}+c \frac{\partial w}{\partial t}+\alpha_{2} C_{n 2} \frac{[V s-V d c]^{2}}{(1+w)^{2}} H_{2}(x) \\
=\alpha_{2} C_{n 1} \frac{[V a c \cos (\Omega t)-V d c]^{2}}{\left(R_{g}-w\right)^{2}} H_{1}(x) \\
+\left[N+\alpha_{1} \int_{0}^{1}\left[\frac{\partial w}{\partial x}\right]^{2} d x\right] \frac{\partial^{2} w}{\partial x^{2}} \\
w(0, t)=w(1, t)=\frac{\partial w}{\partial x}(0, t)=\frac{\partial w}{\partial x}(1, t)=0
\end{array}
$$




\section{Solving}

The beam total displacement $w(x, t)$ can be written as a sum of a static $d c$ displacement $w_{s}(x)$ and a time-varying $a c$ displacement $w_{d}(x, t)$. However, for our devices, it is easy to check that the static deflexion is negligible. Typically, the measured quality factors $Q$ are in the range of $10^{4}-5.10^{4}$ and the $V d c \leq 200 \mathrm{Vac}$. Thus, the ratio between the static and the dynamic deflexion is $\frac{w_{s}(x)}{w_{d}(x, t)} \approx \frac{V d c}{2 Q . V a c} \leq 1 \%$.

A reduced-order model is generated by modal decomposition, transforming Equation (7) into a finite-degree-of-freedom system consisting in ordinary differential equations in time. The undamped linear mode shapes of the straight microbeam are used as basis functions in the Galerkin procedure, and the deflection is approximated by

$$
w(x, t)=\sum_{k=1}^{n} a_{k}(t) \phi_{k}(x)
$$

where $n$ is the number of retained modes (size of the modal basis), $a_{k}(t)$ is the $k^{t h}$ generalized coordinate and $\phi_{k}(x)$ is the $k^{t h}$ linear undamped mode shape of the straight microbeam, normalized such that $\int_{0}^{1} \phi_{k} \phi_{j} d x=0$ for $k \neq j$ and governed by:

$$
\begin{array}{r}
\frac{d^{4} \phi_{k}(x)}{d x^{4}}=\lambda_{k}^{2} \phi_{k}(x) \\
\phi_{k}(0)=\phi_{k}(1)=\phi_{k}^{\prime}(0)=\phi_{k}^{\prime}(1)
\end{array}
$$

Here, $\lambda_{k}$ is the $k^{\text {th }}$ natural frequency of the microbeam. Multiplying Equation (7) by $\phi_{k}(x)\left[(1+w)\left(R_{g}-w\right)\right]^{2}$, substituting Equations (9) and (10) into the resulting equation, and then integrating the outcome from $x=0$ to $x=1$ yields a system of coupled ordinary differential equations in time.

Assuming that the first mode should be the dominant mode of the system and neglecting the terms related to the higher order modes, the following equation is obtained

$$
\begin{array}{r}
\ddot{a}_{1}+\omega_{n}{ }^{2} a_{1}+\mu_{1} a_{1} \ddot{a}_{1}+\mu_{2} a_{1}{ }^{2} \ddot{a}_{1}+\mu_{3} a_{1}{ }^{3} \ddot{a}_{1}+\mu_{4} a_{1}{ }^{4} \ddot{a}_{1} \\
+c \dot{a}_{1}+c \mu_{1} a_{1} \dot{a}_{1}+c \mu_{2} a_{1}{ }^{2} \dot{a}_{1}+c \mu_{3} a_{1}{ }^{3} \dot{a}_{1}+c \mu_{4} a_{1}{ }^{4} \dot{a}_{1} \\
+\chi_{2} a_{1}{ }^{2}+\chi_{3} a_{1}{ }^{3}+\chi_{4} a_{1}{ }^{4}+\chi_{5} a_{1}{ }^{5}+\chi_{6} a_{1}{ }^{6}+\chi_{7} a_{1}{ }^{7} \\
+v+\zeta_{0} \cos (\Omega t)+\zeta_{1} a_{1} \cos (\Omega t)+\zeta_{2} a_{1}{ }^{2} \cos (\Omega t) \\
+\zeta_{3} \cos (2 \Omega t)+\zeta_{4} a_{1} \cos (2 \Omega t)+\zeta_{5} a_{1}{ }^{2} \cos (2 \Omega t)=0
\end{array}
$$

Some canonical nonlinear terms can be identified in Equation (12), such as the cubic stiffness term (Duffing non linearity), the nonlinear Van der Pol damping $\left(c \mu_{2} a_{1}{ }^{2} \dot{a}_{1}\right)$ as well as the parametric excitation (Mathieu term). Other terms correspond to high-level nonlinearities and multifrequency parametric excitations. All these terms come from the coupling between the mechanical and the electrostatic nonlinearities as well as the nonlinear coupling between both electrostatic forces.

To analyse this equation of motion, it proves convenient to invoke perturbation techniques which work well with the assumptions of "small" excitation and damping $(Q>10)$, typically valid in MEMS resonators.

A detuning parameter is introduced as $\Omega=\omega_{n}+\varepsilon \sigma$ and the averaging method [13] is used in order to transform Equation (12) into two first order non-linear ordinary-differential equations which describe the amplitude and phase modulation of the response and permit a stability analysis

$$
\begin{array}{r}
\dot{A}=\varepsilon \frac{\sin [\beta] \zeta_{0}}{2 \omega_{n}}+\varepsilon \frac{A^{2} \sin [\beta] \zeta_{2}}{8 \omega_{n}}-\varepsilon \frac{A c}{2} \\
-\varepsilon \frac{A^{3} c \mu_{2}}{8}-\varepsilon \frac{A^{5} c \mu_{4}}{16}+O\left(\varepsilon^{2}\right) \\
\dot{\beta}=\varepsilon \sigma-\varepsilon \frac{3 A^{2} \chi_{3}}{8 \omega_{n}}-\varepsilon \frac{5 A^{4} \chi_{5}}{16 \omega_{n}}-\varepsilon \frac{35 A^{6} \chi_{7}}{128 \omega_{n}}-\varepsilon \frac{\cos [\beta] \zeta_{0}}{2 A \omega_{n}} \\
-\varepsilon \frac{3 A \cos [\beta] \zeta_{2}}{8 \omega_{n}}+\varepsilon \frac{3}{8} A^{2} \omega_{n} \mu_{2}+\varepsilon \frac{5}{16} A^{4} \omega_{n} \mu_{4}+O\left(\varepsilon^{2}\right)
\end{array}
$$

The steady-state motions occur when $\dot{A}=\dot{\beta}=0$, which corresponds to the singular points of Equations (13) and (14).Thus, the frequency-response equation can be written in its parametric form $\left\{A=K_{1}(\beta), \Omega=K_{2}(\beta)\right\}$ in function of the phase $\beta$.

The critical amplitude is the oscillation amplitude $A_{c}$ above which bistability occurs. The latter can be calculated using the model and assuming that the mechanical nonlinearities are preponderant.

$$
A_{c}=1.685 \frac{h}{\sqrt{Q}}
$$

All the numerical simulations and the experimental characterization were carried out for one of ours devices with the following set of parameters : $l=200 \mu m, b=4 \mu m, h=2 \mu m, l_{a}=20 \mu m$, $g_{a}=1 \mu \mathrm{m}, l_{d}=112 \mu \mathrm{m}, V_{s}=0 \mathrm{~V}$, stress $=15 \mathrm{MPa} . V_{a c}, V_{d c}$ and $g_{d}$ were used for parametric studies.

This analytical model enables the capture of all the non linear phenomena in the resonator dynamics and describes the competition between the hardening and the softening behaviors. In addition, the model allows the optimization of resonator designs by tuning the parameter $\frac{h}{g_{d}}$ in order to cancel out the nonlinearities as shown in Figure 2 for $g_{d}=500 \mathrm{~nm}$ (black curve) and thus enhancing the limit of detection of MEMS and NEMS resonant sensors. 

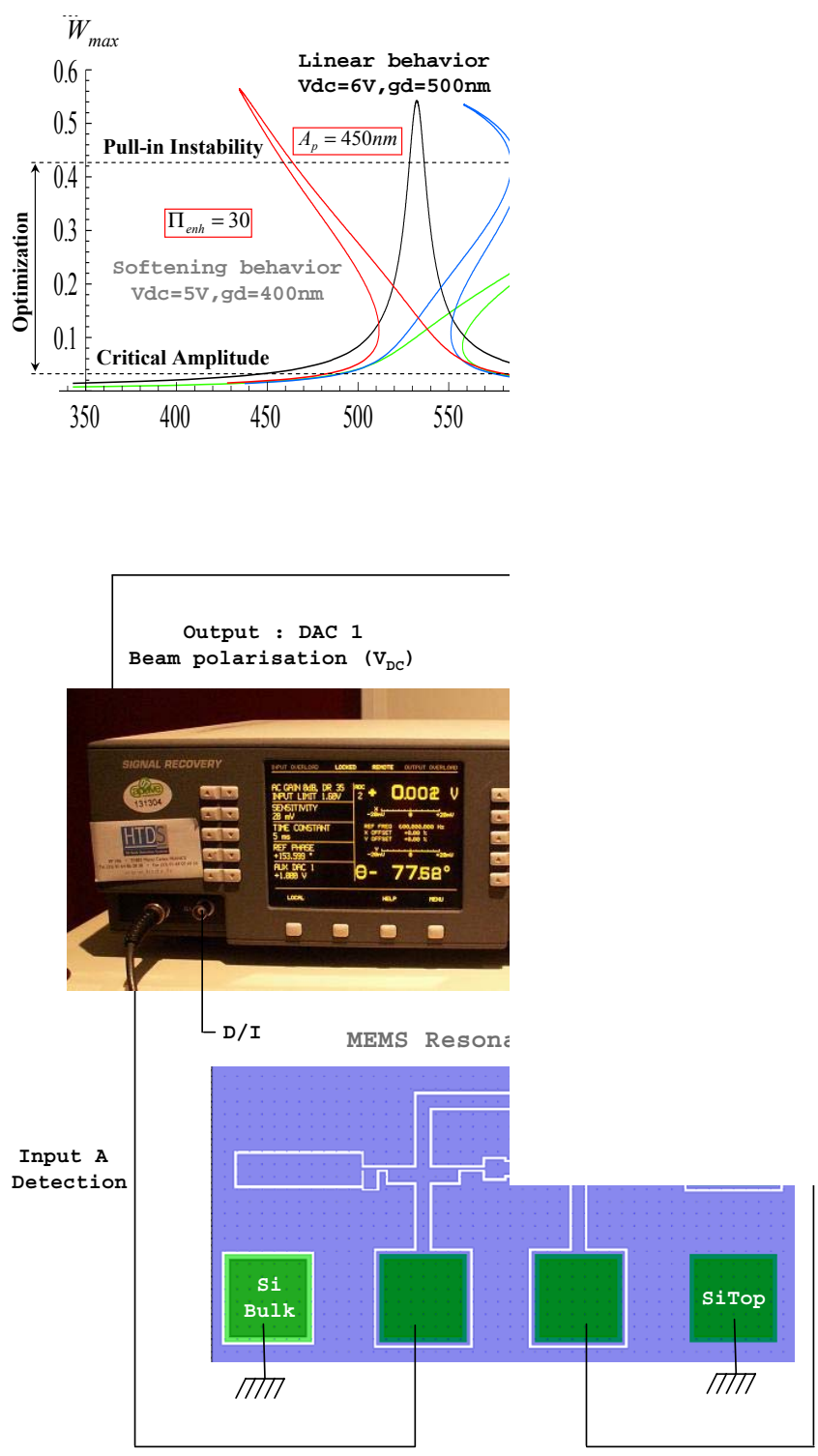

Figure 3. Connection layout for the electrical characterization

\section{Experimental characterization}

The fabricated resonators are electrostatically actuated inplane and their measured quality factors are very high $\left(10^{4}-\right.$ $5.10^{5}$ ). As a consequence, the critical amplitude is around $15 \mathrm{~nm}$ and thus, the capacitance variation is around $2 \mathrm{aF}$. Considering the low capacitance variations [14] and the high motional resistance combined with the important parasitic capacitances, tracking the resonance peak purely electrically is rather awkward. Being at the limit of electric direct measurement, a SEM set-up was developed as a first step, coupled with a real-time in-situ electrical measurement using an external low noise lock-in amplifier (Figure 3).
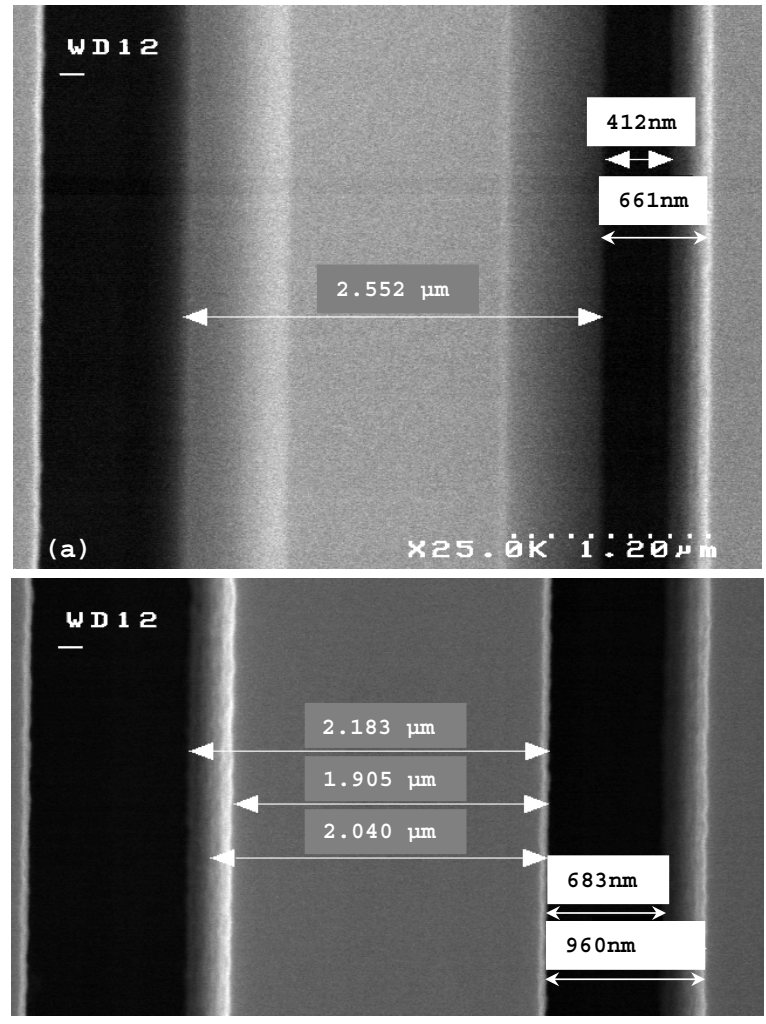

Figure 4. (a): SEM image of the resonator resonance. (b): SEM image of the resonator at rest. Dimensions: $200 \mu m \times 2 \mu m \times 4 \mu m$.

This set-up allows the simultaneous visualization of the resonance by SEM imaging (Figure 4) and the motional current frequency response measurement.

As a second step, once the resonance frequency was found, the SEM setup was not used to permit precise measurements and the device was placed in a vacuum chamber and measurements were performed at room temperature. The residual stress $\left(\frac{\tilde{N}}{b h}\right)$ calculated knowing the frequency shift between the natural frequency and the measured frequency is around $15 \mathrm{MPa}$ and the fringing field effect coefficients are $C_{n 1}=1.6$ and $C_{n 2}=1.5$. As shown in Figure 5, the raw signal given by the lock-in amplifier shows a weak resonance peak drowned in a large background, followed by an antiresonance, both due to a large feedthrough capacitance. To get rid of this effect, a measurement is carried out with null $D C$ voltage. In this case, the beam does not resonate and thus, no motional signal is measured. The vectorial 


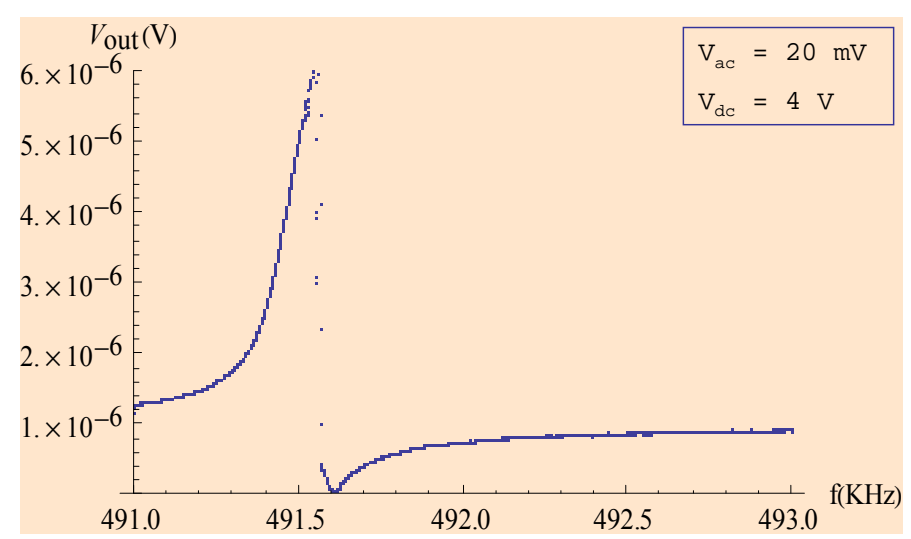

Figure 5. The raw signal given by the lock-in amplifier

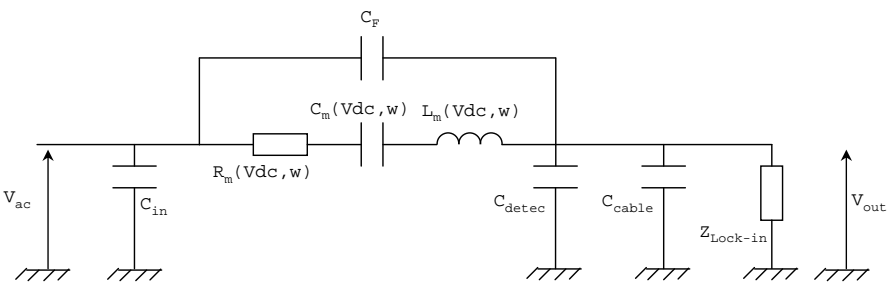

Figure 6. Equivalent electric circuit

subtraction of the two signals gives the signal purely due to the motional current which is compared with the model results.

Considering the equivalent electrical scheme of the measurement chain (see Figure 6), the output voltage generated by this system can be expressed as:

$$
\begin{array}{r}
V_{\text {out }}(t)=\frac{Z_{\text {cable }} Z_{\text {Lockin }}}{Z_{\text {cable }}+Z_{\text {Lockin }}}(V d c-V s) \frac{d C_{\text {res }}}{d t} \\
\frac{d C_{\text {res }}}{d t}=\int_{\frac{l-l_{d}}{2 l}}^{\frac{l+l_{d}}{2 l}} \frac{b C_{n 2} \varepsilon_{0} \phi_{1}(x) a_{1}^{\prime}(t)}{\left(1-a_{1}(t) \phi_{1}(x)\right)^{2}} d x
\end{array}
$$

Where $V_{s}$ is the $D C$ voltage applied to the sensing electrode, $Z_{\text {Lockin }}$ is the internal impedance of the lock-in amplifier and $Z_{\text {cable }}$ is the impedance of the parasitic capacitances due to the connection cables.

The output voltage was calculated analytically using the results of the reduced order model in displacement (Equations (16) and (17)) and a Taylor series expansion of the capacitance. All results shown below were obtained with the same device using the same experimental conditions, and in particular at low enough a pressure so that the quality factor has reached saturation. Only the bias and drive voltages may vary as indicated on the graphs.

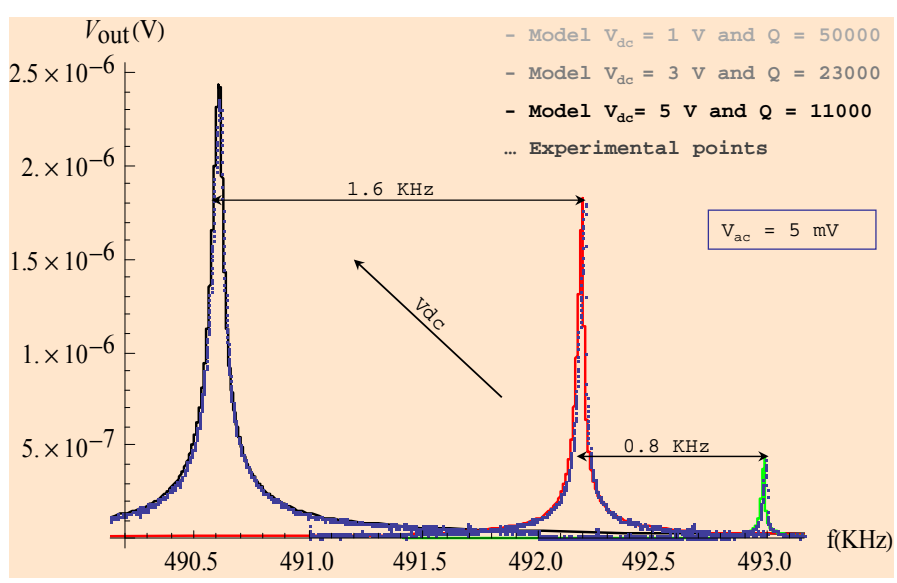

Figure 7. Measured and predicted frequency responses

Linear case $\left(A<A_{c}\right)$ :

The vibration amplitude of the resonator is lower than the critical amplitude. It is paradoxically a difficult condition to obtain, as it demands a low drive, and thus the signals are very weak. A great effort has been needed on the noise and output capacitances reduction to get the peaks out of the background.

It is important to underline that all the inputs of the model are known physical parameters including the fringing field coefficients computed using the analytical formulas [15], except the quality factor $Q$ measured experimentally. No parameter has been tuned to fit the experimental results, in order to assess the prediction capability of the model.

Figure 7 shows 3 linear peaks obtained for different values of the bias voltage $\mathrm{Vdc}(1 \mathrm{~V}-3 \mathrm{~V}-5 \mathrm{~V})$ and same drive voltage. The reader will note that the loaded quality factor change $\left(5.10^{4}-2.3 .10^{4}-1.1 .10^{4}\right)$ accordingly [16]. The resonance frequencies also decrease from $493 \mathrm{KHz}$ (Green curve) to 490.5 $\mathrm{KHz}$ (Black curve) due to the negative stiffness, phenomenon very well displayed by the model. Precisely, the effect of the negative electrostatic stiffness gives frequency shifts of $0.8 \mathrm{KHz}$ between the green and the red curves and $1.6 \mathrm{KHz}$ between the red and the black curve. Moreover, the shape of the peaks and their predicted amplitudes using the model are in excellent correlation with the experimental measured points. Both red and black linear peaks are in the same range of oscillation amplitude (1.8 $\mathrm{V}$ for the red peak and $2.4 \mathrm{~V}$ for the black curve). However, the red peak with high quality factor $(Q=23000)$ is very close to the critical amplitude $\left(V_{\text {out }}=1.9 \mathrm{~V}\right)$, which is well in agreement with Equation (15).

Nonlinear case $\left(A>A_{c}\right)$ :

The vibration amplitude of the resonator is higher than the critical amplitude. The actuation voltage Vac is increased from 


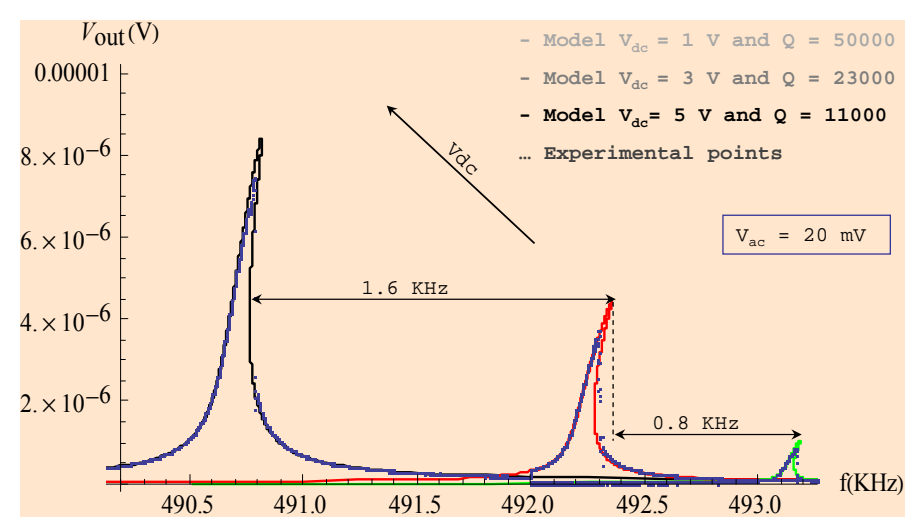

Figure 8. Measured and predicted frequency responses

$5 m V$ used for linear peaks to $20 m V$ here. Figure 8 shows 3 non linear peaks, again obtained for different values of $\mathrm{Vdc}$ $(1 V-3 V-5 V)$. The use of the same resonator, same vacuum conditions and same bias values as in the linear case allows for the identification of the quality factors from the measurements in Figure 7, assuming that no extra damping mechanism takes place. The predicted curves using the model are in very good correlation in shape and frequency shift (negative stiffness) with the measured points, although the model displays slightly higher amplitudes; the unstable jumps make it awkward to obtain precise comparison of high quality factor peaks. Indeed, it is easy to fit perfectly the experimental curves with slightly different values of width, quality factor and residual stress. This is confirmed by the fact that the ratio between the critical amplitude calculated using the model and the peak amplitude measured experimentally $\frac{V_{\text {out }}}{V_{c}}$ is around 5 for the red curve (for which the discrepancy is highest) and 3 for the green and the black curves. Consequently, the red peak is more non linear than the 2 other peaks which is clearly shown in Figure 8 from the curvature of each peak. Also, this validates the close form expression of the critical amplitude (Equation (15)).

\section{Conclusion}

This work has detailed the development of an analytical model and its validation to quantitatively assess the non linear dynamics of M/NEMS resonators. This model includes all sources of non linearities, in particular of the electrostatic ones without approximation and is based on the modal decomposition using the Galerkin procedure combined with a perturbation technique (the averaging method). It has been chosen here to study clamped-clamped beams, but this research will be extended in the future to other types of boundary conditions and structures.

Experimental validation has been performed thanks to the fabrication and electrical measurements of M/NEMS resonators, driven at different (linear and non linear) conditions. All parameters of the model except the quality factor are set prior to the comparison, which shows an excellent agreement in frequency, peak shape and amplitude, although the latter is slightly higher in the model results for highly non linear resonances, which will be further studied. This shows nonetheless it can be an efficient predictive tool.

It has shown how it is possible to tune some design parameters (like the ratio between the beam vibrating width $h$ and the detection gap thickness $g_{d}$ ) to keep a linear behavior up to the pull-in point. The consequence of this may be a great gain in sensors' sensitivity, as the resonator's carrier power is largely increased while keeping linear may prevent most of noise aliasing.

\section{REFERENCES}

[1] Huang, X.M.H., Zorman, C.A., Mehregany, M. and Roukes, M.L, 2003, Nature $\mathbf{4 2 1} 496$

[2] Peng, H.B., Chang, C.W., Aloni, S., Yuzvinsky, T.D., and Zettl, A., 2006, Phys. Rev. Lett. 97087203

[3] Postma, H.W.C., Kozinsky, I., Husain, A., and Roukes, M.L., 2005, Appl. Phys. Lett. 86, 223105

[4] Roukes, M.L., 2001, Nanoelectromechanical systems face the future, Phys. World 14 25-31

[5] Robins, W.P., 1982, Phase Noise in Signal Sources Peter Peregrinus LTD

[6] Juillard, J., Alain, B., Avignon, A., Hentz, S., Kacem, N., and Colinet, E., 2008, Proc. Int. Conf. IEEE Sensors, (Lecce : Italie) 510-513

[7] Kaajakari, V., Koskinen, J.K., and Mattila, T., 2005, IEEE Trans. Ultrason. Ferroelectr. Freq. Control 52 2322-2331

[8] Tilmans, H.A., and Legtenberg, R., 1994, Sensors Actuators A 45 67-84

[9] Gui, C., Legrenberg, R., Tilmans, H.A., Fluitman, J.H.J., and Elwenspoek, M., 1998, J. Microelectromech. Syst. 7 $122-127$

[10] Kozinsky, I., Postma, H.W.Ch., Bargatin, I., and Roukes, M.L., 2006, Appl. Phys. Lett. 88253101

[11] Najar, F., Choura, S., Abdel Rahman, E.M., El-Borgi, S., and Nayfeh, A.H., 2006, J. Micromech. Microeng 162449 2457

[12] Nayfeh, A.H., Younis, M.I., Abdel-Rahman, E.M., 2007, J. Nonlinear Dynamics 48 153-163

[13] Nayfeh, A.H., 1981, Introduction to Perturbation Techniques Wiley (New York)

[14] Truitt, P.A., Hertzberg, J.B., Huang, C.C., Ekinci, K.L., and Schwab, K.C., 2006, Nano Letters 7 (1) 120-126

[15] Nishiyama, H., and Nakamura, M., 1990, IEEE Trans. Comp., Hybrids, Manuf: Technol. 13 417-423

[16] Sazonova, V., 2006, A tunable carbon nanotube resonator, Ph.D. Dissertation, Cornell University (Ithaca, New York) 